\title{
ESTUDO DA ABLAÇÃO EM SUPERFÍCIES DE METAIS IRRADIADAS COM LASER DE FEMTOSSEGUNDO*
}

\author{
Alessandro Francelino Nogueira ${ }^{1}$ \\ Ricardo Elgul Samad ${ }^{2}$ \\ Nilson Dias Vieira Junior ${ }^{3}$ \\ Milton Sergio Fernandes de Lima $^{4}$ \\ Wagner de Rossi $i^{5}$
}

\section{Resumo}

A utilização de lasers de pulsos ultracurtos é uma alternativa para a realização de microusinagem em superfícies de metais, com aplicações diversas em inúmeros segmentos industriais, além das áreas aeronáutica, aeroespacial e naval, onde há crescente preocupação com a eletrônica embarcada. Neste trabalho foram realizadas microusinagens em superfícies de titânio utilizando laser de pulsos ultracurtos de femtossegundos. Tal processo resultou em mínima transferência de calor para o material, evitando assim e deformação superficial da chapa de titânio e a formação de material ressolidificado na região ablacionada, que são inconvenientes presentes na utilização do laser chaveado de pulsos longos da ordem de nanossegundos. Foram executados três tipos de microusinagens, com variações nas distâncias entre as linhas usinadas. Foi verificado ainda que a molhabilidade aumenta quando há o aumento da distância entre linhas usinadas. Finalmente, para a alteração da superfície com uma ínfima remoção de material, constatou-se que a utilização de lasers de pulsos ultracurtos proporcionam grandes benefícios para a integridade do material ablacionado.

Palavras-chave: Ablação de Superfície; Microusinagem; Metais; Laser de Femtossegundo..

\section{STUDY OF ABLATION ON METAL SURFACES IRRADIATED WITH FEMTOSSECOND LASER}

\section{Abstract}

The use of ultrashort pulsed lasers is an alternative for micro-machining on metal surfaces, with diverse applications in several industrial segments, besides aeronautical, aerospace and naval areas, where there is a growing concern with embedded electronics. In this work, micro-machinations were performed on titanium surfaces using ultrashort femtosecond pulses. Such a process resulted in minimal heat transfer to the material, thus avoiding and surface deformation of the titanium plate and the formation of resolidified material in the ablated region, which are drawbacks present in the use of the long pulsed keyed laser of the order of nanoseconds. Three types of micro-machining were performed, with variations in the distances between the machined lines. It was also verified that the wettability increases when there is an increase in the distance between machined lines. Finally, to change the surface with a very small removal of material, it has been found that the use of ultra-short pulse lasers provide great benefits for the integrity of the ablated material.

Keywords: Surface Ablation; Micro-machining; Metal; Femtosecond Laser.

1 Engenheiro Mecânico, Mestre, Doutorando em Tecnologia Nuclear - Materiais, Centro de Lasers e Aplicações, Instituto de Pesquisas Energéticas e Nucleares e Faculdade de Engenharia de Sorocaba - FACENS, São Paulo, SP, Brasil.

2 Físico, Doutor, Pesquisador, Centro de Lasers e Aplicaçbões, Instituto de Pesquisas Energéticas e Nucleares, São Paulo, SP, Brasil. 
3 Físico, Doutor, Pesquisador, Centro de Lasers e Aplicações, Instituto de Pesquisas Energéticas e Nucleares, São Paulo, SP, Brasil.

4 Físico, Doutor, Pesquisador, Instituto de Estudos Avançados, Departamento de Ciência e Tecnologia Aeroespacial, São José dos Campos, SP, Brasil.

5 Físico, Doutor, Pesquisador, Centro de Lasers e Aplicações, Instituto de Pesquisas Energéticas e Nucleares, São Paulo, SP, Brasil. 


\section{INTRODUÇÃO}

A microusinagem, que já fora somente objeto de pesquisas no campo científico, tem atualmente aplicações em diversos segmentos industriais, tais como indústria aeroespacial e aeronáutica, equipamentos médicos, microeletrônica, automotiva, além de utilização em micromotores, circuitos microfluídicos, micro sistemas eletro mecânicos, dispositivos médicos, ferramentas eletrônicas, filtros de partículas, micromoldes e microválvulas, entre outros [1]. Ligas de titânio são amplamente empregadas em elementos estruturais embarcados, por isto a microusinagem com laser é uma importante alternativa para que se consiga alterar as características superficiais, tais como molhabilidade, adesão e cor. Sendo o titânio um material de elevada resistência específica, são empregadas chapas de pequenas espessuras como partes de elementos estruturais, sendo assim, é de suma importância que os processos de microusinagem sejam controlados e não causem danos ao material.

Um importante fator que influencia a precisão na microusinagem a laser é a zona termicamente afetada (ZTA), que se estende além da região de interação do pulso laser e é responsável por uma transformação de fase alterando as propriedades do material colateralmente. O efeito colateral termodinâmico é intrínseco ao processo de ablação quando são utilizados pulsos mais longos que aproximadamente 1 picossegundo, sendo este o caso de lasers comuns, que têm largura temporal da ordem de dezenas de nanossegundos. Contudo este fator pode ser minimizado depositando-se a energia de forma rápida, através de pulsos curtos e ultracurtos, ablacionando assim o material antes que ocorra a difusão do calor e afete a vizinhança da região de interação [2, 3].

Uma das principais características destes pulsos, quando são usados para a microusinagem, é a pequena ZTA, devido à dinâmica particular de absorção e ablação gerada por esses pulsos ultracurtos e ultraintensos. O processo de interação da radiação com a matéria envolve muitos fenômenos que ocorrem em diferentes escalas de tempo, desde femtossegundos até nanossegundos [4]. Os pulsos ultracurtos da ordem de femtossegundos permitiram uma interação determinística com um limiar de ablação bem definido, porém, o limiar de ablação não depende apenas das características do pulso laser e do tipo de material ablacionado. A absorção do pulso também depende da presença de defeitos na estrutura cristalina desses materiais. A pré-irradiação por um pulso laser num material, mesmo que abaixo do limiar de dano, pode criar defeitos na rede cristalina. Esse processo é chamado de efeito de incubação e é responsável pela diminuição do limiar de dano em materiais pré-irradiados. Desta forma, pulsos laser que atingem o material pré-irradiado têm uma maior facilidade na sequência da ablação do material [5].

O processo microusinagem superficial de materiais exige a sobreposição de pulsos para que a quantidade de material necessária seja arrancada. Assim o limiar de ablação e o parâmetro de incubação são parâmetros importantes para a microusinagem e devem ser previamente conhecidos. Nesse contexto, este trabalho teve seu foco na realização de experimentos impondo alterações nas variáveis fluência, taxa de repetição e parâmetros de incubação pela sobreposição de pulsos.

Para obter a condição de mínima transferência de calor para o material, parâmetros adequados de processo precisam ser utilizados. Assim, a fluência deve estar acima do limiar de ablação para o material. Portanto, o início do trabalho concentrou-se na obtenção dos regimes de baixa e de alta fluência, para posteriormente microusinagem com parâmetros de processo adequados ao material. Para a medida 
do limiar de ablação e o parâmetro de incubação, foi utilizada a técnica experimental Diagonal Scan (D-Scan), sendo esta técnica introduzida pelo Centro de Lasers e Aplicações do IPEN, apresentada em [6].

A técnica D-Scan é uma alternativa de método simples para medir o limiar de ablação por pulsos ultracurtos, consistindo em percorrer uma amostra longitudinalmente e transversalmente nas direções $z$ e $y$, conforme mostra a Figura 1(a), através da cintura do feixe focado, a partir de uma posição antes da cintura. Assim, conforme ilustra a Figura 1(b) um perfil simétrico com dois lóbulos é gravado na superfície da amostra.

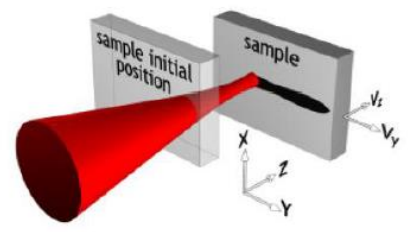

(a)

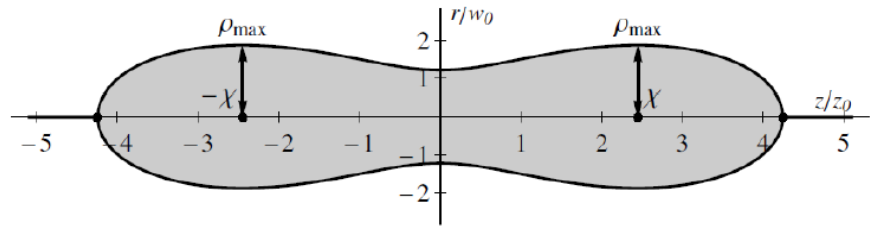

(b)

Figura 1. a) Esquema da técnica D-Scan; b) perfil gravado na superfície da amostra pelo movimento diagonal em posição através da cintura do feixe

O laser de femtossegundo apresenta uma largura temporal muito mais curta que o tempo de interação elétron-fonon, e por este motivo, uma grande quantidade de material pode ser ablacionada antes que a maior parte da energia do feixe de luz seja transferida para a rede cristalina e aqueça o material.

A utilização de lasers para usinagens em metais até então utilizada baseia-se em lasers chaveados de Nd:YAG (Neodymium-doped yttrium aluminium garnet) com pulsos de largura temporal de algumas dezenas de nanossegundos. Neste caso, contudo, o calor produzido pela interação com os pulsos laser causa uma deformação muito grande quando da utilização de chapas muito finas. Assim, o laser de femtossegundo disponível nos laboratórios do Centro de Lasers e Aplicações do Instituto de Pesquisas Energéticas e Nucleares (CLA-IPEN) é uma importante e considerável alternativa para se obter tais superfícies ablacionadas sem a produção colateral de calor.

\section{MATERIAIS E MÉTODOS}

O material escolhido para o este trabalho foi o titânio grau 2 comercialmente puro (Ti Gr. 2 CP) devido sua alta resistência relativa, porém os estudos podem ser ampliados para diversas outras ligas metálicas, uma vez que a metodologia é análoga.

\subsection{Laser de pulsos ultracurtos e sistema de entrega do feixe}

O sistema consiste em um laser de Ti:Safira de femtossegundo amplificado pelo método de amplificação por varredura de frequência (Femtopower Compact Pro CEPhase HP/HR da marca Femtolasers) que gera continuamente pulsos de 25 fs (FWHM) centrados em $775 \mathrm{~nm}$ com $40 \mathrm{~nm}$ de largura de banda (FWHM), taxa de repetição máxima de $4 \mathrm{kHz}$ e energias máxima por pulso de $750 \mu \mathrm{J}$.

O sistema de movimentação para a execução das usinagens consiste em uma mesa com movimento coordenado dos eixos $\mathrm{X}$ e $\mathrm{Y}$ via comando Controle Numérico 
Computadorizado (CNC), enquanto que o movimento no $Z$ é manual através de um micrômetro. Para todos os eixos os movimentos têm precisão micrométrica.

Para estimativa da fluência, considera-se o modelo de propagação de um feixe gaussiano para o cálculo do valor teórico do diâmetro do feixe na superfície da amostra. Desta forma, o diâmetro teórico do feixe na amostra foi determinado conforme a Eq. (1) [7] Siegman (1986).

$\Phi=\frac{4 \cdot M^{2} \cdot \lambda \cdot f}{\pi \cdot \Phi_{0}}$

onde $\boldsymbol{\phi}$ é o diâmetro do feixe no foco, $\boldsymbol{M}^{2}$ é o fator de qualidade do feixe, $\boldsymbol{\lambda}$ é o comprimento de onda do laser, $\boldsymbol{f}$ é o comprimento focal da lente e $\boldsymbol{\phi}_{0}$ é o diâmetro do feixe na entrada da lente. Uma vez determinado o diâmetro teórico do feixe na superfície da amostra, a fluência da energia por pulso é calculada pela razão entre a energia do feixe pela área do feixe no foco $\left(\boldsymbol{F}_{0}=E / \boldsymbol{A f}\right)$.

\subsection{Determinação do limiar de ablação pelo método D-Scan}

O método D-Scan foi realizado em uma amostra de Ti Gr. 2 CP com dimensões de $10 \mathrm{~mm}$ x $20 \mathrm{~mm}$ e espessura de $2,0 \mathrm{~mm}$. No método foram realizados 28 traços, com frequências de $100 \mathrm{~Hz}, 500 \mathrm{~Hz}$ e $4.000 \mathrm{~Hz}$, energias variando de $67,2 \mu \mathrm{J}$ até $71,2 \mu \mathrm{J}$, sobreposição de 0,24 até $7.950,50$ pulsos. Foi utilizado o gás inerte argônio (Ar) com pureza superior a 99,999\% com vazão de 8,0 I/min para proteção da atmosfera das regiões ablacionadas.

Através do Microscópio Eletrônico de Varredura (MEV) foram capturadas as imagens de cada perfil ablacionado e assim medido a metade da dimensão transversal de cada um dos 28 perfis gravados, para que desta forma pudesse ser traçado o gráfico $F_{\text {th }} \times D^{2}$.

\subsection{Análises de Microscopia Eletrônica de Varredura (MEV), Difratometria de raios-X e Molhabilidade}

As análises de MEV foram feitas em um microscópio modelo TM3000 marca Hitachi. A difratometria de raios- $x$ (DRX) foi realizada utilizando o difratômetro de raios- $x$ marca Rigaku modelo Multiflex a $40 \mathrm{kV}$ e $20 \mathrm{~mA}$ usando radiação de Cu-ka $(\lambda=1,54184 \AA$ ). Foram selecionadas a amostra 3 da microusinagem 01 ( $F 0=23,2$ $\mathrm{J} / \mathrm{cm} 2)$ e a amostra 3 da microusinagem $03(\mathrm{FO}=3,3 \mathrm{~J} / \mathrm{cm} 2)$ para realização das análises. A medição da molhabilidade foi realizada pelo método do ângulo de contato da gota séssil, conforme a norma ASTM D 5725-99, utilizando uma pipeta com controle de volume micrométrico da marca HTL modelo LM10 e um arranjo que consiste em um conjunto ótico com uma câmera CMOS marca Thorlabs modelo DCC1545C de resolução $1280 \times 1024$ pixels, acoplada a um sistema de zoom com magnificação variável de até 12 vezes.

\section{RESULTADOS E DISCUSSÃO}

\subsection{Limiar de ablação pelo método D-Scan}

No método D-Scan, foram realizados 28 traços, cujos perfis gravados podem ser vistos na Fig. (1), assim como o gráfico da fluência em função do número de pulsos 
$\left(\boldsymbol{F}_{\text {th }} \boldsymbol{x} \boldsymbol{N}\right)$ para determinação do limiar de ablação para os regimes de baixa e alta fluência, mostrado na Figura 3.

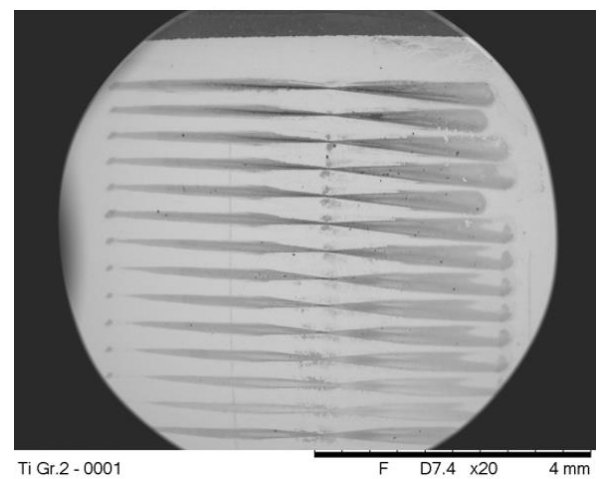

Figura 2. Imagem de MEV dos traços ablacionados na amostra de Ti Gr. 2 CP pelo método D-Scan com aumento de $20 x$

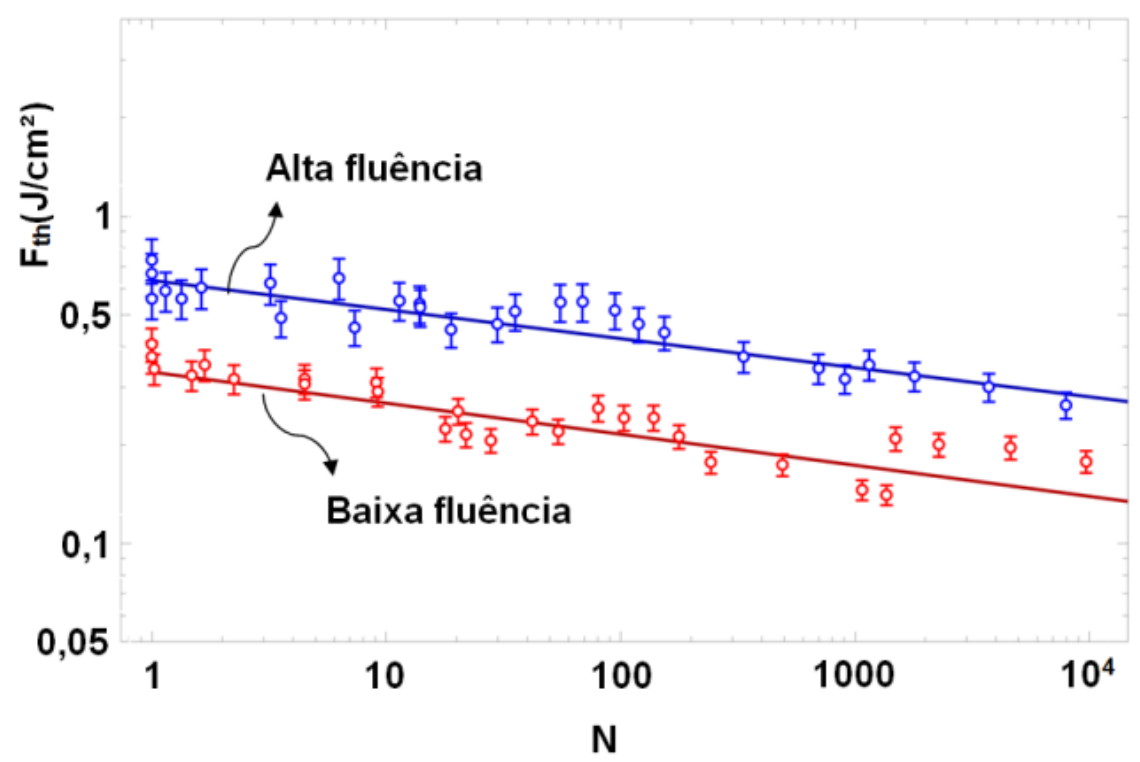

Figura 3. Gráfico da fluência $F_{\text {th }}$ em função do número de pulsos $\mathrm{N}$ sobrepostos para o método $\mathrm{D}$ Scan.

Do gráfico da Fig. (1) tem-se que o limiar de ablação para o regime de baixa fluência para 1 pulso é $F_{\text {th }}=0,330 \pm 0,006 \mathrm{~J} / \mathrm{cm}^{2}$, e para o regime de alta fluência é $F_{\text {th }}=$ $0,640 \pm 0,006 \mathrm{~J} / \mathrm{cm}^{2}$.

\subsection{Microusinagem em lâminas de Ti Gr. 2 CP com espessura de $0,1 \mathrm{~mm}$ utilizando laser de pulsos ultracurtos}

$\mathrm{Na}$ determinação do limiar de ablação pelo método D-Scan, as amostras tinham a espessura de $2 \mathrm{~mm}$. Percebeu-se a não ocorrência de material ressolidificado nas regiões ablacionadas, mesmo no regime de alta fluência. Desta forma, foram executadas 6 (seis) microusinagens em lâminas com espessura de $0,1 \mathrm{~mm}$, utilizando valores de fluência desde $0,44 \pm 0,04 \mathrm{~J} / \mathrm{cm}^{2}$ (microusinagem 1), que está próximo ao limiar, até $18,35 \pm 0,04 \mathrm{~J} / \mathrm{cm}^{2}$ (microusinagem 6), com o intuito de atestar a ausência de empenamento. A sobreposição de pulsos foi de 50 para a microusinagem 1 e reduzida para 1,144 na microusinagem 2 , sendo que da microusinagem 3 até a microusinagem 6 não houve sobreposição, ou seja, os 
pontos ablacionados ficaram espaçados. Em todos os casos foi utilizado o gás inerte argônio (Ar) com pureza superior a 99,999\% com vazão de 8,0 l/min para proteção da atmosfera. Vale aqui salientar, que o diâmetro utilizado para estes cálculos foi o diâmetro teórico do feixe laser focalizado sobre a amostra conforme Erro! Fonte de referência não encontrada. que foi de $24 \mu \mathrm{m}$. Para fluências maiores, os danos são maiores e o espaçamento precisa ser maior.

$\mathrm{Na}$ microusinagem 1, que tem a maior sobreposição, o efeito de incubação promoveu a maior redução no limiar de ablação, sendo a condição onde ocorreu o maior aporte térmico. As microusinagens 1, 2 e 4 podem ser vistas em imagens de MEV na Fig. (3), onde pode ser constatada a sobreposição de pulsos para o primeiro caso e o distanciamento dos pulsos nos demais.

À medida que é aumentada a sobreposição, a relação entre o tamanho do dano e o diâmetro teórico do pulso no foco aumenta, o que é a consequência direta do efeito de incubação. Pode-se notar ainda que na microusinagem 2 a sobreposição foi mínima, ou seja, com uma sobreposição de 14,4\% somente. Devido à combinação dos parâmetros frequência e velocidade, nas microusinagens 3, 4, 5 e 6 não houve sobreposição e desta forma foram obtidas trilhas de pontos para estes casos.

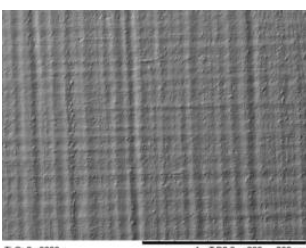

(a)

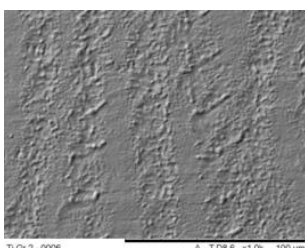

(b)

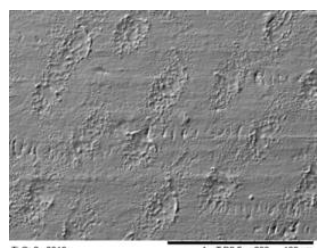

(c)

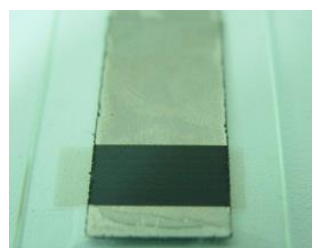

(d)

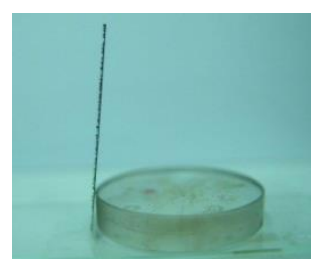

(e)

Figura 4. Imagens de MEV a) na lâmina de $0,1 \mathrm{~mm}$ de espessura da microusinagem 1 com aumento de $300 x$, b) da microusinagem 2 com aumento de $1.000 x, c)$ e da microusinagem 4 com aumento de $800 x$. Fotografia d) da lâmina com a microusinagem 1 vista de topo e e) da vista de perfil.

Mesmo na condição extrema, que foi a microusinagem 1, não ocorreram deformações da lâmina, comprovando que mesmo no regime de alta fluência não há significativa transferência de calor à rede cristalina que pudesse causar tais deformações. A Fig. (3)(e) mostra a lâmina com esta microusinagem, podendo ser constatado que não houve qualquer indício de deformação superficial que causasse empenamento e distorções.

Contudo, nas microusinagens onde não ocorreu a sobreposição de pulsos, a usinagem dos furos cegos ("dimples") necessitaria de um tempo extremamente alto para sua execução. Isto acarreta que haveria a necessidade de repetidos passes na mesma região para que ocorresse sobreposição para garantir uma boa profundidade nos pontos ablacionados. Desta forma, a realização de "dimples" pelo sistema disponível seria inviável, o que direcionou os trabalhos para uma outra estratégia, que seria a realização de "traços em malhas" para a obtenção das microusinagens.

Como a matriz de pontos produzidos no processo anterior levou a danos muito rasos, foram definidas três microusinagens com maior fluência de energia por pulso e sobreposição consideravelmente maior que a da microusinagem 1, objetivando a microusinagem de traços com uma grande sobreposição de pulsos e a produção de sulcos profundos. As três microusinagens nas lâminas de $0,1 \mathrm{~mm}$ de espessura foram usinadas utilizando o gás inerte argônio com pureza superior a 99,999\% com vazão de 8,0 l/min para proteção da atmosfera.

Para a confecção das microusinagens optou-se por executar uma microusinagem em zig-zag cruzada na lâmina de $0,1 \mathrm{~mm}$. Para a execução destas geometrias em zig-zag cruzado somente houve a variação do passo entre as linhas usinadas, sendo 
de $75 \mu \mathrm{m}$ para a microusinagem 01 , de $100 \mu \mathrm{m}$ para a microusinagem 02 e de 200 $\mu \mathrm{m}$ para a microusinagem 03. A fluência de energia por pulso foi de $3,32 \pm 0,04$ $\mathrm{J} / \mathrm{cm}^{2}$ e a sobreposição foi de 1150 pulsos para as 3 (três) microusinagens.

Após a execução das microusinagens foram feitas análises em MEV, cujas imagens obtidas estão ilustradas na Fig. (4). Assim, foi analisado e constatado que mesmo nesta condição extrema da geometria em zig-zag, ou seja, uma condição onde foi promovida uma considerável remoção de material com sensível profundidade não houve deformação da lâmina.

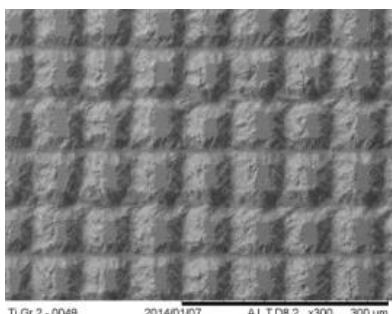

(a)

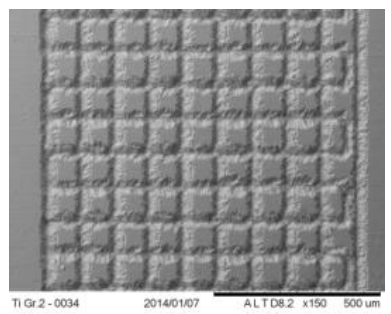

(b)

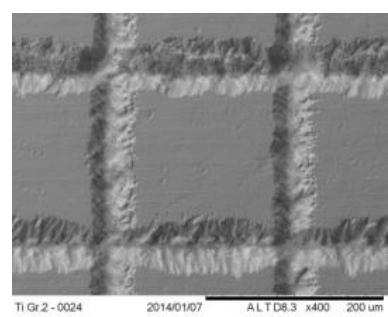

(c)

Figura 5. Imagens de MEV das microusinagens com geometrias zig-zag cruzadas em lâmina de 0,1 $\mathrm{mm}$ de espessura. a) Microusinagem $01 \mathrm{com}$ aumento de 300x; b) microusinagem 02 com aumento de 150x; e c) microusinagem 03 com aumento de 400x.

São mostradas as imagens da microusinagem 01 em três dimensões obtidas no MEV com o auxílio do software 3D-Image Viewer na Fig. (5), onde pode ser notado que a lâmina de $0,1 \mathrm{~mm}$ de espessura não sofreu deformações e empenamento. Pela análise da topografia foi possível medir a profundidade das regiões microusinadas, que resultou em um valor médio de 14,50 $\mu \mathrm{m}$.
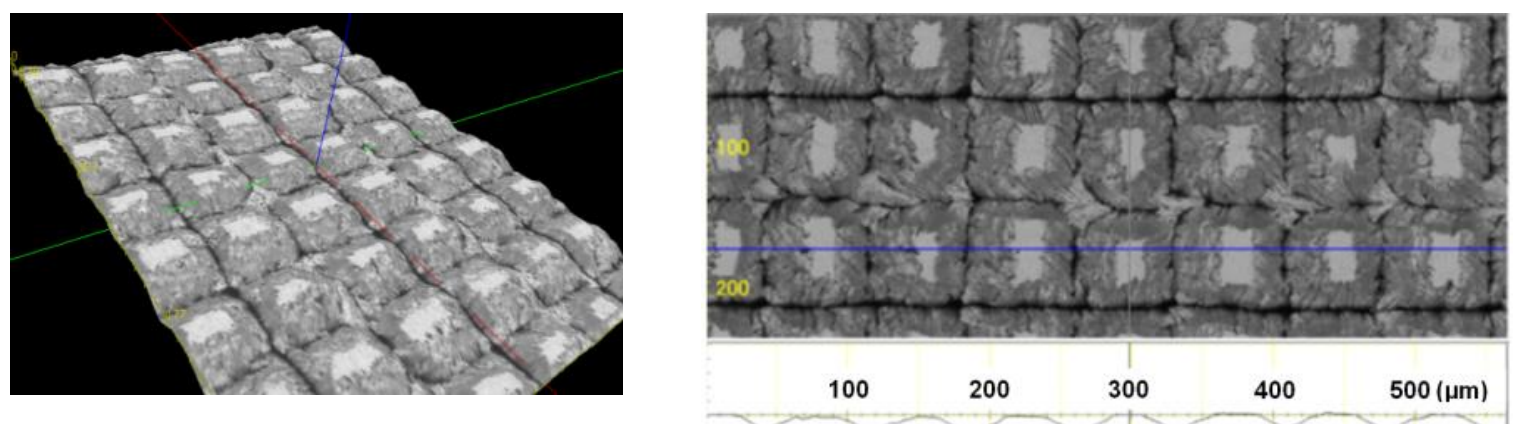

Figura 6. Imagens de MEV em três dimensões da microusinagem em zig-zag 01 através do software 3D-Image Viewer com aumento de 300x.

Os parâmetros utilizados para a confecção destas microusinagens proporcionaram uma condição bastante favorável em virtude de haver uma sobreposição considerável de pulsos e ter promovido uma profundidade satisfatória, além de não ter sido evidenciada a existência de material ressolidificado e tampouco apresentado indícios de empenamento. Com estas condições, esperava-se que a colagem teria uma boa eficiência.

A Figura 7 apresenta imagem de MEV com linhas feitas na determinação do limiar de ablação pelo método D-Scan, onde é possível ver a região afetada pelo regime de baixa fluência, que é a região vizinha à linha usinada. Estas linhas foram executadas com fluência de energia por pulso e sobreposição similares às condições em que foram realizadas as microusinagens. 


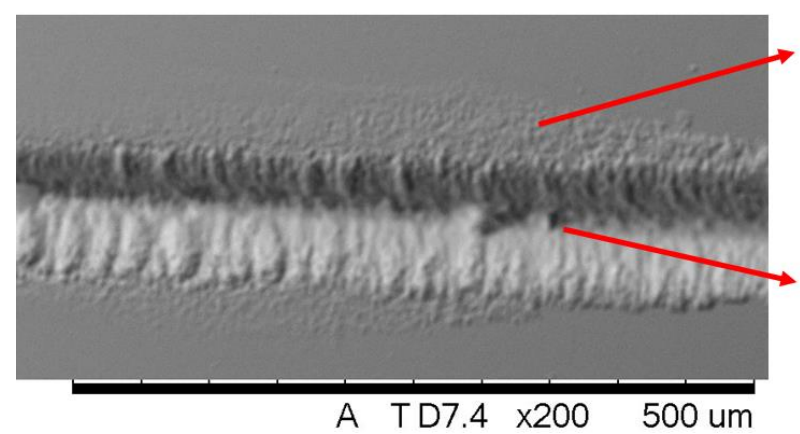

Região vizinha

afetada pelo regime

de baixa fluência

Linha texturizada - no

centro da linha ocorre

a máxima fluência

FIGURA 7. Imagem de MEV de linha realizada no método D-Scan com fluência de energia por pulso e sobreposição similares às condições das microusinagens. Aumento de 200x.

\subsection{Microusinagem em amostras de $2 \mathrm{~mm}$ de espessura}

A partir da conclusão de que seria possível atuar no regime de alta fluência sem que houvesse deformações nas amostras, foram então microusinadas amostras com 2 $\mathrm{mm}$ de espessura. Isto foi necessário, pois para os casos das lâminas de $0,1 \mathrm{~mm}$ não seria possível a realização de ensaio de tração. Foram realizadas microusinagens em 3 (três) amostras da microusinagem 01, 6 (seis) amostras da microusinagem 02 e 6 (seis) amostras da microusinagem 03 com os mesmos parâmetros do caso anterior.

As dimensões das amostras são de $60 \mathrm{~mm} \times 10 \mathrm{~mm}$ e espessura de $2 \mathrm{~mm}$, e a área microusinada foi de $10 \mathrm{~mm}$ x $10 \mathrm{~mm}$ na extremidade das amostras. Para proteção da atmosfera foi utilizado o gás inerte argônio com pureza superior a 99,999\% e vazão de $8,0 \mathrm{l} / \mathrm{min}$.

\subsection{Difratometria de raios-X (DRX) em amostras de $2,0 \mathrm{~mm}$ de espessura microusinadas}

Pelas análises de difratometria de raios- $X$ das amostras microusinadas, foi verificado que não há fase $\beta$ (estrutura cristalina cúbica de corpo centrado) retida, uma vez que somente foi constatada a presença do Ti somente em sua fase a (estrutura cristalina hexagonal compacta) - Powder Diffraction File PDF 05-0682. Vale ressaltar que a técnica utilizada tem sensibilidade para detectar em uma camada de até 1 (um) $\mu \mathrm{m}$, desta forma se houvesse a presença de fase $\beta$, ela estaria em uma camada menor que 1 (um) $\mu \mathrm{m}$. Assim, de forma evidente, no processo de ablação por laser de pulsos ultracurtos não houve a presença de fase $\beta$ após o resfriamento.

Mesmo atuando no regime de alta fluência, e que acarretou formação de fase líquida, não se nota material ressolidificado. Isto provavelmente ocorreu porque o material fundido foi ejetado para fora da região afetada, e não teve tempo (ou energia suficiente) para aquecer a região vizinha e provocar uma zona afetada pelo calor detectável. Assim, mesmo com alta fluência, a estrutura cristalina da superfície não é modificada e, portanto não gera tensões que levariam à deformação da peça.

Contudo, como o titânio possui alta afinidade com o oxigênio, pode-se verificar a presença de TiO na amostra com a microusinagem 01 (Powder Diffraction File PDF 89-3077) e na amostra com a microusinagem 03 (Powder Diffraction File - PDF 71-5272), mesmo com proteção da atmosfera de interação com gás inerte argônio. Desta forma, pela extrema reatividade do titânio com o oxigênio, mesmo com a utilização do argônio ainda assim houve a reação com o oxigênio e a consequente 
formação de óxidos. Uma forma de se evitar ou minimizar esta reação seria a utilização de uma "glove box" com baixíssima concentração de oxigênio.

\subsection{Medição da molhabilidade}

As imagens dos perfis das gotas estão apresentadas na Fig. (6), e com o auxílio do software ImageJ, Figura 9, foram determinados os ângulo de contato conforme ilustrado.

As medições dos ângulos de contato indicaram os valores de $99,85^{\circ}, 83,56^{\circ}$ e $50,08^{\circ}$ para as microusinagens 01,02 e 03 respectivamente, enquanto que para a superfície sem microusinagem resultou no valor de $39,54^{\circ}$. Os resultados da molhabilidade para cada amostra indicam o decréscimo do ângulo de contato com o aumento do passo de microusinagem, o que aumentou a hidrofilia da superfície e consequentemente melhorou a molhabilidade. Ainda pode-se perceber que a amostra sem microusinagem apresenta a melhor molhabilidade.

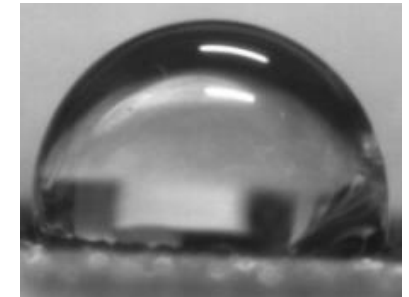

(a)

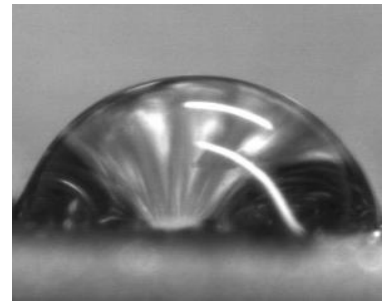

(b)

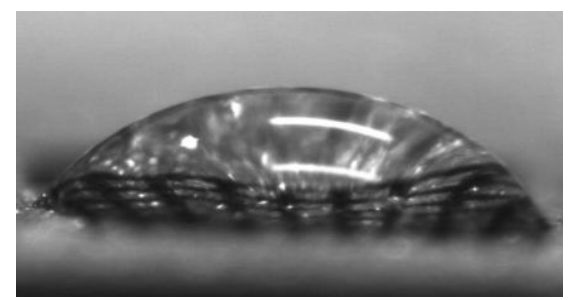

(c)

Figura 8. Imagem do perfil da gota para medição da molhabilidade pelo método da gota séssil na a) microusinagem 01, b) microusinagem 02, c) microusinagem 03.

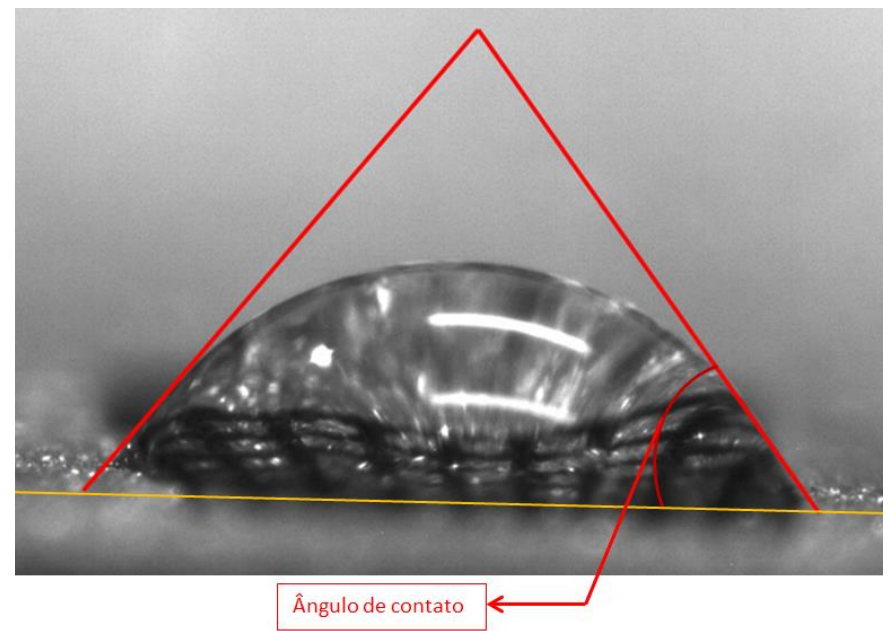

Figura 9. Imagem da microusinagem 03 no software Image $\mathrm{J}$ - linhas representam as referências para determinação do ângulo de contato da gota.

É importante salientar que neste experimento, apesar de o método utilizado seguir as recomendações da ASTM D 5725-99, não foi utilizado um equipamento específico para medição de molhabilidade, e desta forma os valores obtidos podem apresentar alguma variação em relação ao que seriam encontrados em tal equipamento, sendo assim a análise quantitativa não oferece confiabilidade quanto aos exatos valores, porém permite analisar as variações para cada geometria 
usinada. Desta forma os valores obtidos tem um caráter muito mais qualitativo para fins de comparação de cada microusinagem realizada do que propriamente para determinação de características de molhabilidade das superfícies.

O efeito imediato da microusinagem da superfície do metal é a influência exercida no ângulo de contato da gota, modificando suas propriedades superficiais.

\section{CONCLUSÃO}

Por meio da obtenção dos limiares de ablação para o Ti Gr. 2, pelo laser de pulsos ultracurtos de femtossegundos, através da realização do experimento pelo método D-Scan foi possível a determinação dos regimes de alta e baixa fluência. Com o sistema utilizado neste trabalho não foi possível obter microusinagens de "dimples" com pulsos laser de femtossegundos, visto que seria extremamente demorado. A alternativa então de se atuar no regime de alta fluência para produzir uma geometria de "traços em malha" mostrou-se eficiente. Através do conhecimento dos limiares de ablação, foram realizadas microusinagens em três condições em lâminas de Ti Gr. 2 $\mathrm{CP}$ de $0,1 \mathrm{~mm}$ de espessura. Tais microusinagens foram realizadas atuando no regime de alta fluência, visto que mesmo nesta condição não acarretou em distorções e empenamento às lâminas além de que o material fundido foi totalmente expelido para fora da região processada, deixando uma superfície livre de material ressolidificado. O tempo de aquecimento, e/ou a energia introduzida não foram suficientes para produzir uma ZTA suficientemente grande para ser detectada, além de ter promovido uma satisfatória profundidade.

A análise das microusinagens realizadas permitiu a mensuração da molhabilidade e dos volumes específicos para cada célula unitária bem como o volume total disponível. Desta forma, foi verificado que quanto menor a molhabilidade, maior é o número de células unitárias e por consequência, maior o volume total disponível.

Desta forma, a utilização de laser de pulsos ultracurtos mostrou-se extremamente satisfatória para a realização de microusinagens em Ti Gr. 2 CP, com a possibilidade de realização de microusinagens com variações nas geometrias e que possibilitam ainda a extensão do estudo à outros metais, mantendo a integridade do material e com ótima qualidade da região ablacionada.

\section{Agradecimentos}

Os autores agradecem à FAPESP (Projeto 2013/26113-6), ao IPEN, ao Centro Tecnológico da Marinha em São Paulo e à Faculdade de Engenharia de Sorocaba (FACENS) pelo apoio e suporte dado ao desenvolvimento desta pesquisa.

\section{REFERÊNCIAS}

1 Samad RE, Machado LM, Vieira ND Jr, De Rossi W. Ultrashort Laser Pulses Machining, In: I. Peshko (Ed.) Laser Pulses - Theory, Technology, and Applications, InTech, 2012;143-174.

2 Jandeleit J, Horn A, Weichenhain R, Kreutz EW, Poprawe R. Fundamental investigations of micromachining by nano- and picosecond laser radiation, Applied Surface Science. 1998;127:885-891.

3 Pronko PP, Dutta SK, Squier J, Rudd JV, Du D, Mourou G. Machining of sub-micron holes using a femtosecond laser at 800 nm", Optics Communications. 1995;114(12):106-110. 
4 Bulgakova NM, Stoian R, Rosenfeld A, Hertel IV, Campbell EEB. Electronic transport and consequences for material removal in ultrafast pulsed laser ablation of materials. Physical Review B- Condensed Matter and Materials Physics, 2004; 69(5):054102.

5 Ashkenasi D, Rosenfeld A, Stoian R. Laser-induced incubation in transparent materials and possible consequences for surface and bulk micro-structuring with ultrashort pulses. Society of Photo-Optical Instrumentation Engineers (SPIE). 2002;4633.

6 Samad RE, Vieira ND Jr. Geometrical method for determining the surface damage threshold for femtosecond laser pulses. Laser Physics. 2006; 16(2):336-339.

7 Siegman AE. Lasers. University Science Books, Salsalito, CA, United States, 1978:1283 p. 